\title{
The Effect of Completeness of Revascularization During CABG with Single versus Multiple
}

\section{Arterial Grafts}

Short Title: Incremental Benefit of functional revascularization

Thomas A. Schwann, $\mathrm{MD}^{1,2}$, Maroun B. Yammine, $\mathrm{MD}^{3}$, Abdul-Karim M. El-Hage-Sleiman, $\mathrm{MD}^{3}$,

Milo C. Engoren, MD ${ }^{2,4}$ Mark R. Bonnell, MD¹ and Robert H. Habib, $\mathrm{PhD}^{3,5}$

${ }^{1}$ University of Toledo College of Medicine and Life Sciences, Toledo, $\mathrm{OH}$, USA

${ }^{2}$ Mercy Saint Vincent Medical Center, Toledo, $\mathrm{OH}$, USA

${ }^{3}$ Department of Internal Medicine, Outcomes Research Unit, and Vascular Medicine Program, American University of Beirut, Lebanon

${ }^{4}$ Department of Anesthesiology, University of Michigan, Ann Arbor, MI, USA

${ }^{5}$ Society of Thoracic Surgery Research Center, Chicago, IL, USA

\section{Corresponding Authors:}

Thomas A. Schwann, MD, MBA

University of Toledo College of Medicine and Life Sciences,

3000 Arlington Ave, Toledo, $\mathrm{OH}, 43615$ USA

Tel: 419-383-5150

Thomas.Schwann@utoledo.edu

Research funded by departmental and Institutional funds. Authors MBY and AME are funded in part on NIH Training grant 1D43TW009118-01A1 awarded to Scholars in Health Research Program, American University of Beirut, Beirut - Lebanon.

All authors have no conflict of interest to disclose

This manuscript has an online supplement

Word Count: 3,839

This is the author manuscript accepted for publication and has undergone full peer review but has not been through the copyediting, typesetting, pagination and proofreading process, which may lead to differences between this version and the Version of Record. Please cite this article as doi: 10.1111 jocs. 13810 


\begin{abstract}
Introduction: Incomplete coronary revascularization is associated with suboptimal outcomes. We investigated the long-term effects of Incomplete, Complete and Supra-complete revascularization and whether these effects differed in the setting of single-arterial and multiarterial CABG.
\end{abstract}

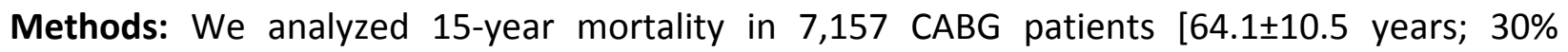
women]. All patients received a left internal thoracic artery to left anterior descending coronary artery graft with additional venous grafts only (single-arterial) or with at least one additional arterial graft (multi-arterial) and were grouped based on a completeness of revascularization index $(C R I=$ number of grafts minus the number of diseased principal coronary arteries: Incomplete [CRI $\leq-1 \quad(\mathrm{~N}=320 ; 4.5 \%)]$; Complete [CRI=0 ( $\mathrm{N}=2882 ; 40.3 \%) ;$ reference group]; and two Supra-complete categories $[C R I=+1(N=3050 ; 42.6 \%) ; C R I \geq+2(N=905 ; 12.6 \%)]$. Risk-adjusted mortality hazard ratios (AHR) were calculated using comprehensive propensity score adjustment by Cox regression.

Results: Incomplete revascularization was rare (4.5\%) but associated with increased mortality in all patients $[\mathrm{AHR}(95 \% \mathrm{Cl})=1.53(1.29-1.80)]$, those undergoing single-arterial CABG $[A H R=1.27(1.04-1.54)$ and multi-arterial $C A B G[A H R=2.18(1.60-2.99)]$ as well as in patients with 3-Vessel $[A H R=1.37(1.16-1.62)]$ and, to a lesser degree, with 2-vessel $[A H R=1.67(0.53-5.23)]$ coronary disease. Supra-complete revascularization was generally associated with 
incrementally decreased mortality in all patients $[A H R(C R I=+1)=0.94(0.87-1.03)$; $\operatorname{AHR}(C R I \geq+2)=0.74(0.64-0.85)]$, and was driven by a significantly decreased mortality risk in single-arterial $C A B G[A H R(C R I=+1)=0.90(0.81-0.99) ; A H R(C R I \geq+2)=0.64(0.53-0.78)]$; and $3-$ Vessel disease patients $[A H R(C R I=+1)=0.94(0.86-1.04)$; and $A H R(C R I \geq+2)=0.75(0.63-0.88)]$ with no impact in multi-arterial CABG $[A H R(C R I=+1)=1.07(0.91-1.26) ; \operatorname{AHR}(C R I \geq+2)=0.93(0.73-1.17)]$

Conclusions: Incomplete revascularization is associated with decreased late survival, irrespective of grafting strategy. Alternatively, supra-complete revascularization is associated with improved survival in patients with 3-Vessel CAD, and in single-arterial but not multiarterial CABG.

Key words: CABG, Completeness of Revascularization, Multi-Arterial grafting, Propensity Score 
Coronary artery bypass grafting (CABG) remains the optimal treatment for multi-vessel coronary artery disease (CAD). ${ }^{1-3}$ Certain surgeon decisions may substantially impact late CABG outcomes. Among these are: 1) the extent to which normal myocardial perfusion is restored, or completeness of revascularization ${ }^{4-12}$ and 2 ) the choice of arterial versus venous conduits as coronary grafts. ${ }^{13-16}$

Incomplete coronary revascularization has been repeatedly associated with worse survival after $C A B G$, leading surgeons to adopt strategies to facilitate complete revascularization. ${ }^{4-6}$ One approach to accomplish complete revascularization focuses on the construction of one graft for each major diseased coronary artery system. ${ }^{7-9}$ Another approach to complete revascularization entails placement of grafts to all diseased coronary vessels supplying viable myocardium, even within the same coronary system. ${ }^{10-12}$ This approach necessarily entails a higher number of grafts, but whether this technique translates to incrementally improved survival has not been systematically assessed. ${ }^{11}$

Concurrently, compared to the standard-of-care single arterial left internal thoracic artery (LITA)-based CABG with only additional saphenous vein grafts (SVG), a number of studies have demonstrated improved late CABG survival with multi-arterial CABG achieved by the additional use of either the right-ITA or radial artery (RA) grafts. ${ }^{13-16}$ Whether multi-arterial grafting modifies the effect of the completeness of revascularization on late survival is not well elucidated. One recent study suggested that multi-arterial grafts mitigated the unfavorable long-term effects of incomplete revascularization, but the investigators did not differentiate between varying levels coronary revascularization. ${ }^{17}$ 
The current study analyzed a large CABG experience in multi-vessel CAD patients to test the hypothesis that compared to complete revascularization, incomplete revascularization is associated with decreased late survival, and that Supra-complete revascularization results in incrementally improved late survival. A secondary aim of this analysis was to examine whether these effects are modified in case of multi-arterial compared to single-arterial CABG.

\section{METHODS}

This study is a retrospective analysis of prospectively collected cardiac surgery databases from two Ohio hospitals [Mercy Saint Vincent Medical Center (1994-2007) and University of Toledo Medical Center (2000-2011), Toledo, Ohio]. Patient data were collected in accordance with the Society of Thoracic Surgeons (STS) Cardiac Surgery Database and did not involve further patient contact or hospital record reviews. Institutional Review Boards from both medical centers approved this study and waived the need for patients' informed consent.

Patients who underwent primary CABG surgery for multi-vessel CAD and received a LITA to left anterior descending coronary artery (LAD) graft were included in the study. Patients were excluded from analysis in case of emergency-salvage, re-operation, no LITA, preoperative renal failure, or in case of concomitant valvular or aortic surgery. We defined completeness of revascularization index $(\mathrm{CRI})$ as the difference between the number of coronary grafts and the number of diseased coronary systems. ${ }^{18}$ Each distal anastomosis was counted as a separate graft. Thus a single sequential conduit counted as more than one graft. Patients were grouped based on their derived CRI: Incomplete $[\mathrm{CRI} \leq-1 \quad(\mathrm{~N}=320 ; 4.5 \%)]$; Complete $[\mathrm{CRI}=0 \quad(\mathrm{~N}=2882$; 
$40.3 \%)$; and two levels of Supra-complete [CRI=+1 ( $N=3050 ; 42.6 \%) ; C R I \geq+2$ ( $N=905 ; 12.6 \%)]$ revascularization.

Surgical Technique. The surgical techniques have been described previously. ${ }^{14,16}$ The radial artery was used in $41 \%$ of the study population. Bilateral internal thoracic artery (BITA) grafting was used infrequently (2.6\%).

Follow-up. The primary outcome was long-term all-cause mortality. Long-term mortality data was obtained from recurrent searches of the US Social Security Death Index (SSDI) database (http://ssdi.genealogy.rootsweb.com), last search November 2011 as this was no longer a valid research tool thereafter. ${ }^{19}$ The available study follow-up period ranged between 3 and 189 months.

\section{Statistical Methods}

Continuous data were presented as mean \pm standard deviation and categorical data as percentages. Univariate comparisons were done with chi-square $\left(X^{2}\right)$ and Student t-test or ANOVA test as appropriate with applied Bonferroni correction. Patient demographics, risk factors and operative variables exhibited significant differences across CRI Cohorts (Table 1), for single-arterial versus multi-arterial grafting (Table S-1; online supplement), as well as the 2vessel and 3-vessel coronary artery disease subgroups (Table S-2; online supplement).

Propensity-score adjustment was used as the main method to account for these imbalances and associated confounding in all analyses. The primary analysis comparing survival outcomes across CRI groups was done on the entire study population. Secondary analyses were 
also done in four specific patient sub-cohorts - each based on a cohort-specific propensity score model: single-arterial CABG, multi-arterial CABG, 2-Vessel disease, and 3-Vessel disease.

For each analysis, a propensity score predicting the probability of $\mathrm{CRI}=0$ (reference category) versus all other sub-groups combined was calculated using a non-parsimonious logistic regression model based on 37 demographic, comorbidity, risk factor, surgical priority and graft type variables (Table 1). Highly collinear variables were avoided. Given the long study period (1994-2011), the year of the surgery was included in the propensity score calculation. The resulting propensity scores for the CRI sub-cohorts were distinctly different in all comparisons. Unadjusted (HR) and propensity score adjusted hazard ratios [AHR (95\% confidence intervals)] were derived by Cox regression analysis assuming proportional hazards. Derived AHRs were confirmed using comprehensive risk adjusted Cox regression including all patient factors in the propensity score models. Predictors of incomplete $(C R I \leq-1)$ and supracomplete revascularization $(C R I=+1$ and $C R I \geq+2)$ were obtained using binary logistic regression with the complete revacularization $(C R I=0)$ group used as the reference category (Backward selection). A p value less than 0.05 was used to indicate statistical significance. Analysis was done using SPSS version 21 software (IBM, Armonk, NY).

\section{RESULTS}

The total study population consisted of 7157 (72\%) multi-vessel CABG patients drawn from the overall CABG population of 9865 performed between 1994 and 2011. Mean age and female sex decreased with increasing CRI (both $p \leq 0.001$ ), while cardiopulmonary bypass times and multi-arterial grafting increased significantly (both $\mathrm{p} \leq 0.001$ ). Ejection fraction, diabetes and 
triple vessel disease were also different among CRI groups (all $p \leq 0.001$ ). Generally, incomplete revascularization (CRI $\leq-1)$ patients had higher prevalence of comorbidities (peripheral vascular disease, cerebrovascular disease, chronic obstructive lung disease; all $p \leq 0.001)$, but incomplete revascularization was not independently associated with a higher operative mortality risk (Table 1). Despite increased time on cardiopulmonary bypass and a longer aortic cross clamp time, higher levels of CRI did not carry an increased acute operative mortality (CRI $\leq-1: 1.9 \%, C R I=0$ : $1.6 \%, \mathrm{CRI}=+1: 1.4 \%, \mathrm{CRI} \geq+2: 0.9 \% ; \mathrm{p}=0.44)$.

Patient and operative factors were also substantially different when compared for the single-arterial $(n=4015 ; 56 \%)$ versus multi-arterial $(n=3142 ; 44 \%)$ sub-cohorts [Table S-1] and for the 2-Vessel disease $(n=1564 ; 22 \%)$ versus 3 -Vessel disease $(n=5593 ; 78 \%)$ sub-cohorts [Table S-2; online supplement]. Multi-arterial CABG patients were younger, more male, with a higher prevalence of 3-Vessel disease and lower prevalence of insulin-dependent diabetes compared to single-arterial counterparts (all $p \leq 0.001$ ). Notably, the distribution of the CRI differed significantly for single-arterial and multi-arterial CABG patients $(p \leq 0.001)$. The most common level of $C R I$ in single-arterial was $C R I=0(45.6 \%)$ followed by $C R I=+1(40.1 \%)$, while this was reversed in multi-arterial $C A B G$ patients $[C R I=0(33.4 \%), \quad C R I=+1(45.8 \%)]$. CABG corresponding to a $\mathrm{CRI} \geq+2$ was performed in $8.2 \%$ of single-arterial and $17.8 \%$ of multi-arterial CABG patients. Increasing levels of CRI was accomplished by increasing both venous and radial

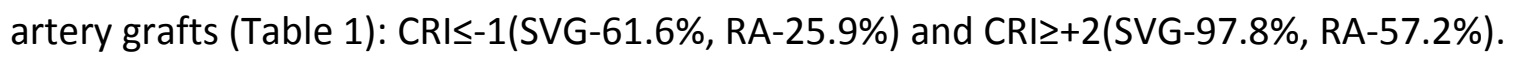

Incomplete revascularization (CRI $\leq-1$ ) was relatively rare, found only in $4.5 \%$ of all patients, and was more frequent among 3 -Vessel disease versus 2 -Vessel disease $(5.5 \%$ vs $1.0 \%$, 
$p<0.001)$ and single-arterial versus multi-arterial CABG patients $(5.6 \%$ vs $3.0 \%, p<0.001)$. Predictors of incomplete revascularization were: female sex [OR(95\% Confidence Interval): $1.39(1.06-1.81)]$, age $\left[1.02(1.00-1.03)\right.$ per year], body surface area $\left[1.69(0.99-2.86)\right.$ per $\left.\mathrm{m}^{2}\right]$, peripheral vascular disease [1.66(1.27-2.17)] and 3-Vessel Disease [5.91(3.50-9.96)]. A majority of patients received Supra-complete revascularization (55.2\%; $C R I=+1$ and $C R I \geq+2$ groups), and these were more frequent among multi-arterial versus single-arterial CABG patients (63.6\% vs 48.7\%; $p<0.001)$, in men [OR(95\% Confidence Interval), 1.66(1.50-1.85], in patients with diabetes [1.15(1.03-1.29); insulin-dependent: 1.31(1.13-1.53)], and less likely in patients with peripheral vascular disease [0.81(0.71-0.92)), cerebrovascular disease [0.80(0.72-0.90)], previous PCI [0.82(0.72-0.92)], and 3-Vessel disease [0.87(0.78-0.98)].

\section{Long-term survival}

Unadjusted survival based on CRI category are shown in Figure 1 (Top, middle, bottom) for the all patients, single-arterial $C A B G$, and multi-arterial CABG cohorts, respectively. Figure 2 shows the unadjusted Kaplan Meier survival comparison for CRI groups in patients with 2Vessel disease and 3-Vessel disease. Generally, Incomplete revascularization (CRI $\leq-1$ ) consistently showed worse survival, compared to complete revascularization (CRI=0), irrespective of arterial grafting strategy or degree of vessel disease. Alternatively, increasing levels of CRI $(+1$, or $\geq+2)$ showed incrementally improved survival, compared to complete revascularization $(C R I=0)$, in case of single-arterial $C A B G$ and 3-Vessel disease but not for multiarterial CABG. The associated unadjusted hazard ratios $( \pm 95 \%$ Confidence Interval) are summarized in Table S-3. 
Figure 3 summarizes the adjusted hazard ratios across the various revascularization categories for all patients, as well as for arterial grafting strategy [Figure 3 (Left)] and Vessel disease [Figure 3 (Right)] subcohorts. These risk-adjusted analyses confirmed the significantly worse survival with incomplete revascularization in essentially all cases: All-Patients $\{[\mathrm{HR}(95 \%$ CI): 1.53(1.29-1.80)], single-arterial CABG [1.27(1.04-1.54)], multi-arterial CABG [2.08(1.522.84)], 3-Vessel Disease [1.37(1.16-1.62)]\}. The 2-Vessel disease cohort was small $(n=15)$ and the trend toward worse survival did not reach statistical significance [1.67(0.53-5.23)]. Notably, despite the small number of patients $(n=94)$, the deleterious effects of incomplete revascularization were more pronounced in case of multi-arterial CABG compared to singlearterial. Increasing $\mathrm{CRI}(\mathrm{CRI}=0,+1$, or $\geq+2)$ showed incrementally decreased mortality in the overall study cohort. This effect of supra-complete revascularization was more substantial and significant in case of single-arterial CABG [CRI=+1: 0.90(0.81-0.99), CRI= $\geq+2: 0.64(0.53-0.78)]$ but not multi-arterial CABG [CRI=+1: 1.07(0.91-1.26), $C R I=\geq+2: 0.93(0.73-1.17)]$. Lastly, increasing levels of CRI was particularly beneficial in patients with 3-Vessel CAD and less so in patients with 2-Vessel CAD [Figure 3].

\section{Discussion}

In this large CABG experience with a substantial use of multiple arterial conduits, our findings confirm that although incomplete revascularization $(C R I \leq-1)$ does not increase perioperative mortality, it is associated with a significantly increased 15-year mortality. Furthermore, increasing the number of coronary grafts to achieve Supra-complete revascularization $(C R I=+1$ and $C R I \geq+2)$, as opposed to complete revascularization $(C R I=0)$, 
results in an incremental systematic decrease in late mortality without any appreciable increase in perioperative risk inherent to increased operative times required to achieve this grafting strategy. This survival benefit of a supra-complete revascularization is particularly evident in case of single-arterial as opposed to multi-arterial CABG, and in patients with 3-Vessel CAD as opposed to 2-Vessel CAD.

A uniformly accepted definition of incomplete coronary revascularization is lacking and hence its impact on late survival is not well defined. Traditionally, incomplete revascularization has been defined by the lack of a graft placed to one or more of the three principal epicardial coronary systems ${ }^{20}$ containing a hemodynamically significant lesion. The lack of a graft to any coronary target with $\geq 50 \%$ stenosis, even within the same coronary artery system (LAD, circumflex or the RCA) has also been considered as an alternative definition of an incomplete revascularization. ${ }^{18}$ Others, using more sophisticated and also more complex criteria, have defined incomplete revascularization based on the residual post procedural myocardial jeopardy index ${ }^{21}$, a decremental deviation from an "ab initio" grafting strategy as formulated in the Syntax Trial ${ }^{22}$ or a residual Syntax score greater than zero. ${ }^{23}$ These varied definitions probably contribute to the wide discrepancies in the reported rates of incomplete revascularization from $5 \%^{24}$ to $36.8^{22}$ to as high as $69 \%^{21}$. We report a relatively low overall incomplete revascularization rate of $4.5 \%$.

Surgeons are generally committed to a complete coronary revascularization aimed at fully restoring myocardial perfusion in all coronary vessels supplying viable myocardial territories. Unforeseen intra-operative factors (diminutive coronary targets, diffuse atherosclerosis, lack of conduits, a porcelain aorta or accepting incomplete revascularization to 
minimize operative time) may lead to modification of such a pre-operative plan resulting in incomplete revascularization. The literature on the clinical impact of incomplete revascularization is split, with some reports indicating significant adverse clinical outcomes $^{5,9,21,24,25}$ while others suggesting a trivial or no impact on clinical end-points. ${ }^{4,17,18,26}$ This is crystallized by the results of two separate analyses of the SYNTAX trial data with one study concluding that incomplete revascularization did not impact outcomes ${ }^{22}$, while a follow up study found incomplete revascularization was associated with adverse outcomes. ${ }^{23}$ Our data demonstrate that incomplete revascularization is associated with increased late mortality in the overall CABG population as well as inpatient sub-cohorts undergoing single-arterial CABG, multi-arterial CABG and in patients with 3-Vessel CAD. This trend did not reach statistical significance in 2-Vessel CAD patients, although given the small number of patients in this group ( $n=15$ cases only) these results must be interpreted with caution and require confirmation from larger analyses. This negative effect of incomplete revascularization was particularly prominent in multi-arterial CABG. Our finding differs from other studies. Kieser et al reported no adverse long-term outcomes associated with incomplete revascularization in patients younger than 80 and who underwent predominantly multi-arterial CABG (98\%). ${ }^{17}$ In contradistinction to our findings, the authors concluded that multi-arterial grafting mitigates the negative impact of incomplete revascularization. In patients older than 80 , however, incomplete revascularization carried an increased risk of mortality but no explanation behind these findings was provided. ${ }^{17}$ We did not study the impact of age on the effects of incomplete revascularization. Rastan et al, in a large CABG series with a $21 \%$ rate of all arterial grafting, found no difference in survival up to 5 years post-operatively between patients with a complete versus an incomplete 
revascularization, although the results were not risk adjusted. ${ }^{27}$ Potential explanations for the different findings in our analysis compared to these other studies include: the rate of incomplete revascularization in our multi-arterial CABG cohort was quite small $(n=94)$, our 16year follow-up was substantially longer, and both the Kieser and Rastan groups had a much higher rate of bilateral internal thoracic artery (BITA) use and a much lower radial artery (RA) use compared to our multi-arterial cohort. Thus conceivably incomplete revascularization in BITA-based multi-arterial CABG may have distinctly different prognosis than in LITA/RA-based multi-arterial CABG.

A number of preoperative factors were found to be associated with incomplete revascularization including female sex, increasing age, increasing body surface area, peripheral vascular disease and 3-Vessel disease. Others have also identified older age, diabetes and peripheral vascular disease as predictors. ${ }^{23}$ Conversely, we identified that male sex [OR (95\% Confidence Interval): 1.66 (1.50-1.85] and diabetes [OR=1.15 (1.03-1.29)] are associated with a greater frequency of supra-complete revascularization (CRI=+1 and $C R I \geq+2)$. Possibly the larger size of coronary targets in males lend themselves to a higher number of grafts, while the more diffuse and aggressive diabetic coronary angiopathy may necessitate more grafts per each diseased coronary artery system.

Our data support a supra-complete revascularization, with placement of a coronary graft to each diseased target vessel rather than a single conduit to each diseased coronary artery system, particularly in cases of single-arterial CABG and in patients with 3-Vessel CAD. Chu et $a^{28}$ compared risk adjusted 10-year outcomes in Veterans Administration CABG patients receiving one conduit per diseased coronary system to those receiving multiple grafts per 
diseased coronary system and found no difference in survival with either grafting strategy. The fact that $99 \%$ of this cohort was male, with a relatively low incidence of triple vessel disease ( $53 \%$ vs $78 \%$ in the current study) and the exclusive use of the LITA as the only arterial graft, may account for the differences in the results. Our finding that a supra-complete revascularization strategy is not associated with an incremental survival benefit compared to Complete revascularization in patients undergoing multi-arterial CABG may be due to the survival benefit associated with the increased durability of arterial grafts compared to venous grafts and thus a smaller number of arterial grafts may have a similar impact on survival as a larger number of venous grafts. Furthermore, slower progression of atherosclerotic burden downstream from arterial grafts compared to venous grafts may also play a role in our findings ${ }^{29}$ suggesting that it is the quality of the grafts rather than quantity of grafts among multi-arterial CABG patients that is important.

The limitations of our study include its retrospective, single practice two institutional nature. Our assessment of coronary disease, consistent with the STS database criteria, is rather rudimentary based on the number of diseased major coronary artery systems without consideration of the specific number of vessels diseased within each system. In addition, our CRI calculation includes the number of completed coronary grafts without consideration of the specific coronary targets. In certain cases, due t our reliance on the derived CRI metrics, a CRI=0 designation, may in fact represent an incomplete revascularization, if the grafts were placed to the same coronary artery system leaving diseased vessel in other coronary artery systems unreconstructed. The limited granularity of our dataset does not permit this level of detailed analysis. Furthermore, our data did not include information on the complexity of the 
atherosclerotic burden, the quality of the target vessels or their size. Also, we are unable to categorize the coronary artery lesions as proximal or distal and how much myocardium remains at risk by not bypassing them. In addition, our database does not contain the reasons why a patient left the operating room with an incomplete revascularization, the details of the physiologic significance of the coronary lesions as the severity of each stenosis was exclusively based on angiographic assessment rather than their impact on physiologic myocardial perfusion or whether a diseased vessel supplied viable or non-viable myocardium. We also do not have an assessment of the collateral flow in patients with an incomplete revascularization. Lastly, the specific cause of death was unavailable to us to specifically assess the cardiovascular consequences of the achieved revascularization.

In conclusion, our data strongly supports the perspective that incomplete revascularization is associated with increased long term mortality regardless of whether a patient undergoes single-arterial CABG or multi-arterial CABG. Furthermore, Supra-complete revascularization ( $C R I=+1$ and $C R I \geq+2)$ compared to complete revascularization $(C R I=0)$, appears to be associated with improved long-term survival in patients undergoing single-arterial CABG and in those with 3-Vessel disease. In multi-arterial CABG, the incremental benefit of additional grafts beyond those required for a complete revascularization is likely of smaller magnitude. Given the small numbers of patients within our study subcohorts, our findings should be considered hypothesis generating and additional, more granular, confirmatory studies are warranted to inform shared decision making by the Heart Team and the patient. 


\section{References}

1. Iqbal J, Serruys PW, Taggart DP. Optimal revascularization for complex coronary artery disease. Nat Rev Cardiol. 2013;10(11):635-647.

2. Osnabrugge RL, Head SJ, Bogers AJ, Kappetein AP. Multivessel coronary artery disease: quantifying how recent trials should influence clinical practice. Expert Rev Cardiovasc Ther. 2013;11(7):903-918.

3. Taggart DP. Lessons learned from the SYNTAX trial for multivessel and left main stem coronary artery disease. Curr Opin Cardiol. 2011;26(6):502-507.

4. Mohammadi S, Kalavrouziotis D, Dagenais F, Voisine $P$, Charbonneau E. Completeness of revascularization and survival among octogenarians with triple-vessel disease. Ann Thorac Surg. 2012;93(5):1432-1437.

5. Kleisli T, Cheng W, Jacobs MJ, et al. In the current era, complete revascularization improves survival after coronary artery bypass surgery. J Thorac Cardiovasc Surg. 2005;129(6):1283-1291.

6. Girerd N, Magne J, Rabilloud M, et al. The impact of complete revascularization on longterm survival is strongly dependent on age. Ann Thorac Surg. 2012;94(4):1166-1172.

7. Bell MR, Gersh BJ, Schaff HV, et al. Effect of completeness of revascularization on longterm outcome of patients with three-vessel disease undergoing coronary artery bypass surgery. A report from the Coronary Artery Surgery Study (CASS) Registry. Circulation. $1992 ; 86(2): 446-457$.

8. Caputo M, Reeves BC, Rajkaruna C, Awair H, Angelini GD. Incomplete revascularization 
during OPCAB surgery is associated with reduced mid-term event-free survival. Ann Thorac Surg. 2005;80(6):2141-2147.

9. Kozower BD, Moon MR, Barner HB, et al. Impact of complete revascularization on longterm survival after coronary artery bypass grafting in octogenarians. Ann Thorac Surg. 2005;80(1):112-116.

10. Ong AT, Serruys PW. Complete revascularization: coronary artery bypass graft surgery versus percutaneous coronary intervention. Circulation. 2006;114(3):249-255.

11. Zimarino M, Curzen N, Cicchitti V, De Caterina R. The adequacy of myocardial revascularization in patients with multivessel coronary artery disease. Int J Cardiol. 2013;168(3):1748-1757.

12. Gossl M, Faxon DP, Bell MR, Holmes DR, Gersh BJ. Complete versus incomplete revascularization with coronary artery bypass graft or percutaneous intervention in stable coronary artery disease. Circ Cardiovasc Interv. 2012;5(4):597-604.

13. Locker C, Schaff HV, Dearani JA, Daly RC. Improved late survival with arterial revascularization. Ann Cardiothorac Surg. 2013;2(4):467-474.

14. Zacharias A, Habib RH, Schwann TA, Riordan CJ, Durham SJ, Shah A. Improved survival with radial artery versus vein conduits in coronary bypass surgery with left internal thoracic artery to left anterior descending artery grafting. Circulation. 2004;109(12):1489-1496.

15. Habib RH, Abou-Arraj NE, Schwann TA. Radial artery as a second arterial graft in the elderly and both sexes. Ann Cardiothorac Surg. 2013;2(4):453-457.

16. Schwann TA, Zacharias A, Riordan CJ, Durham SJ, Shah AS, Habib RH. Sequential radial 
artery grafts for multivessel coronary artery bypass graft surgery: 10-year survival and angiography results. Ann Thorac Surg. 2009;88(1):31-39.

17. Kieser TM, Curran HJ, Rose MS, Norris CM, Graham MM. Arterial grafts balance survival between incomplete and complete revascularization: a series of 1000 consecutive coronary artery bypass graft patients with $98 \%$ arterial grafts. J Thorac Cardiovasc Surg. 2014;147(1):75- 83.

18. Vander Salm TJ, Kip KE, Jones RH, et al. What constitutes optimal surgical revascularization? Answers from the Bypass Angioplasty Revascularization Investigation (BARI). J Am Coll Cardiol. 2002;39(4):565-572.

19. Blackstone EH. Demise of a vital resource. J Thorac Cardiovasc Surg. 2012; 143:37-38.

20. McNeer JF, Conley MJ, Starmer CF, et al. Complete and incomplete revascularization at aortocoronary bypass surgery: experience with 392 consecutive patients. Am Heart J. 1974;88(2):176-182.

21. Schwartz L, Bertolet M, Feit F, et al. Impact of completeness of revascularization on long-term cardiovascular outcomes in patients with type 2 diabetes mellitus: results from the Bypass Angioplasty Revascularization Investigation 2 Diabetes (BARI 2D). Circ Cardiovasc Interv. 2012;5(2):166-173.

22. Head SJ, Mack MJ, Holmes DR, Jr., et al. Incidence, predictors and outcomes of incomplete revascularization after percutaneous coronary intervention and coronary artery bypass grafting: a subgroup analysis of 3-year SYNTAX data. Eur J Cardiothorac Surg. 2012;41(3):535-541.

23. Farooq V, Serruys PW, Bourantas CV, et al. Quantification of incomplete 
revascularization and its association with five-year mortality in the synergy between percutaneous coronary intervention with taxus and cardiac surgery (SYNTAX) trial validation of the residual SYNTAX score. Circulation. 2013;128(2):141-151.

24. Ngaage DL, Hashmi I, Griffin S, Cowen ME, Cale AR, Guvendik L. To graft or not to graft? Do coronary artery characteristics influence early outcomes of coronary artery bypass surgery? Analysis of coronary anastomoses of 5171 patients. J Thorac Cardiovasc Surg. $2010 ; 140(1): 66-72$

25. Scott R, Blackstone EH, McCarthy PM, et al. Isolated bypass grafting of the left internal thoracic artery to the left anterior descending coronary artery: late consequences of incomplete revascularization. J Thorac Cardiovasc Surg. 2000;120(1):173-184.

26. Moon MR, Sundt TM, 3rd, Pasque MK, Barner HB, Gay WA, Jr., Damiano RJ, Jr. Influence of internal mammary artery grafting and completeness of revascularization on longterm outcome in octogenarians. Ann Thorac Surg. 2001;72(6):2003-2007.

27. Rastan AJ, Walther T, Falk V, et al. Does reasonable incomplete surgical revascularization affect early or long-term survival in patients with multivessel coronary artery disease receiving left internal mammary artery bypass to left anterior descending artery? Circulation. 2009;120(11 Suppl):S70-77.

28. Chu D, Bakaeen FG, Wang XL, Coselli JS, LeMaire SA, Huh J. The impact of placing multiple grafts to each myocardial territory on long-term survival after coronary artery bypass grafting. J Thorac Cardiovasc Surg. 2009;137(1):60-64.

29. Dimitrova KR, Hoffman DM, Geller CM, et al. Arterial grafts protect the native coronary vessels from atherosclerotic disease progression. Ann Thorac Surg 2012; 94: 475-81. 


\section{Figure Legends}

Figure 1. Comparison of 15-year survival for the four completeness of revascularization (CRI subgroups) shown for the overall cohort (top: All CABG) and separately for the single arterial (middle: single-arterial CABG) and multi-arterial (bottom: multi-arterial CABG) sub-cohorts. P value reflects the long rank test across all 4 groups (overall).

Figure 2. Comparison of 15-year survival for the four completeness of revascularization (CRI subgroups) shown for the 2-vessel disease (top) and 3-vessels disease (bottom) patient subcohorts. P value reflects the long rank test across all 4 groups (overall).

Figure 3. Propensity score adjusted hazard ratios for 15 -year mortality following CABG surgery shown for different completeness of revascularization levels ( $C R I=0$ as reference): (Left) overall cohort (All CABG) and separately for the single arterial (single-arterial CABG) and multi-arterial (multi-arterial CABG) sub- cohorts; (Right) overall cohort (All CABG) and separately for the 2vessel disease and 3-vessel disease sub-cohorts. 
Table 1. Selected patient demographic, co-morbidity and operative data compared across Completeness of Revascularization (CRI) Subcohorts 
Table 1. Selected patient demographic, co-morbidity and operative data compared across Completeness of Revascularization (CRI) Subcohorts.

\begin{tabular}{|c|c|c|c|c|c|}
\hline \multirow[b]{2}{*}{ Variable } & \multicolumn{4}{|c|}{ Completeness of revascularization Index } & \multirow[b]{2}{*}{$p$-value *,\# } \\
\hline & $\begin{array}{c}\text { CRI } \leq-1 \\
(N=320)\end{array}$ & $\begin{array}{c}C R I=0 \\
(N=2882)\end{array}$ & $\begin{array}{c}C R I=+1 \\
(N=3050)\end{array}$ & $\begin{array}{c}C R I \geq+2 \\
(N=905)\end{array}$ & \\
\hline Categorical Variables & $\%$ & $\%$ & $\%$ & $\%$ & \\
\hline Female & $34.7 \%$ & $36.2 \%$ & $28.2 \%$ & $17.2 \%$ & $\leq 0.001$ \\
\hline BMI $\left(\mathrm{Kg} / \mathrm{m}^{2}\right)$ & & & & & 0.274 \\
\hline$<25$ & $16.3 \%$ & $17.8 \%$ & $16.4 \%$ & $13.5 \%$ & \\
\hline $25-29.9$ & $36.6 \%$ & $38.4 \%$ & $39.1 \%$ & $38.8 \%$ & \\
\hline $30-34.9$ & $29.4 \%$ & $27.2 \%$ & $27.8 \%$ & $28.2 \%$ & \\
\hline $35-39.9$ & $10.9 \%$ & $10.7 \%$ & $11.0 \%$ & $13.4 \%$ & \\
\hline$>40$ & $6.9 \%$ & $5.9 \%$ & $5.7 \%$ & $6.2 \%$ & \\
\hline Smoking & $57.5 \%$ & $62.4 \%$ & $61.3 \%$ & $61.2 \%$ & 0.374 \\
\hline Family History of CAD & $66.6 \%$ & $65.5 \%$ & $64.5 \%$ & $62.2 \%$ & 0.278 \\
\hline Diabetes & $36.6 \%$ & $34.5 \%$ & $35.8 \%$ & $40.0 \%$ & 0.029 \\
\hline Insulin Dependent & $15.9 \%$ & $10.9 \%$ & $12.3 \%$ & $11.5 \%$ & 0.043 \\
\hline Hypercholesterolemia & $69.1 \%$ & $71.9 \%$ & $70.2 \%$ & $71.3 \%$ & 0.415 \\
\hline Hypertension & $85.0 \%$ & $81.0 \%$ & $80.3 \%$ & $78.7 \%$ & 0.086 \\
\hline Peripheral Vascular Disease & $24.7 \%$ & $17.5 \%$ & $14.8 \%$ & $13.7 \%$ & $\leq 0.001$ \\
\hline Cerebrovascular Disease & $30.3 \%$ & $25.2 \%$ & $21.4 \%$ & $17.8 \%$ & $\leq 0.001$ \\
\hline Stroke & $10.9 \%$ & $8.3 \%$ & $7.4 \%$ & $6.0 \%$ & 0.17 \\
\hline COPD & $25.0 \%$ & $20.5 \%$ & $20.0 \%$ & $15.7 \%$ & $\leq 0.001$ \\
\hline Myocardial Infarction & $58.1 \%$ & $52.7 \%$ & $55.4 \%$ & $53.8 \%$ & 0.089 \\
\hline
\end{tabular}




\begin{tabular}{|c|c|c|c|c|c|}
\hline Congestive Heart Failure & $12.2 \%$ & $11.5 \%$ & $10.9 \%$ & $9.8 \%$ & 0.466 \\
\hline Previous PCl & $20.6 \%$ & $19.7 \%$ & $17.5 \%$ & $16.9 \%$ & 0.056 \\
\hline Three Vessels Disease & $95.3 \%$ & $77.2 \%$ & $78.6 \%$ & $73.5 \%$ & $\leq 0.001$ \\
\hline Left Main Disease & $19.4 \%$ & $23.8 \%$ & $22.6 \%$ & $21.7 \%$ & 0.218 \\
\hline \multicolumn{6}{|l|}{ Operative Variables } \\
\hline Emergency & $5.3 \%$ & $5.1 \%$ & $5.6 \%$ & $5.9 \%$ & 0.754 \\
\hline ON-Pump Surgery & $96.6 \%$ & $97.4 \%$ & $97.9 \%$ & $96.1 \%$ & $\leq 0.001$ \\
\hline Non isolated CABG & $10.3 \%$ & $9.1 \%$ & $8.8 \%$ & $10.8 \%$ & 0.508 \\
\hline Radial artery graft use & $25.9 \%$ & $33.6 \%$ & $44.5 \%$ & $57.2 \%$ & $\leq 0.001$ \\
\hline Saphenous Vein graft use & $61.6 \%$ & $87.5 \%$ & $96.8 \%$ & $97.8 \%$ & $\leq 0.001$ \\
\hline $\begin{array}{l}\text { Bilateral Internal Mammary } \\
\text { Artery }\end{array}$ & $3.4 \%$ & $2.6 \%$ & $2.1 \%$ & $3.9 \%$ & 0.026 \\
\hline Peri-op Mortality & $1.9 \%$ & $1.6 \%$ & $1.4 \%$ & $0.9 \%$ & 0.440 \\
\hline Continuous Variables & Mean \pm SD & Mean $\pm S D$ & Mean $\pm S D$ & Mean士SD & $p$-value $*, \#$ \\
\hline Age (years) & $66.0 \pm 10.7$ & $64.6 \pm 10.9$ & $63.9 \pm 10.4$ & $62.6 \pm 10.0$ & $\leq 0.001$ \\
\hline Body Surface area (BSA, $\mathrm{m}^{2}$ ) & $2.03 \pm 0.25$ & $2.01 \pm 0.25$ & $2.04 \pm 0.25$ & $2.09 \pm 0.24$ & $\leq 0.001$ \\
\hline Ejection Fraction (\%) & $48 \pm 11$ & $50 \pm 11$ & $49 \pm 11$ & $48 \pm 11$ & $\leq 0.001$ \\
\hline Cardio-pulmonary Bypass (min) & $49 \pm 38$ & $68 \pm 30$ & $90 \pm 31$ & $112 \pm 33$ & $\leq 0.001$ \\
\hline Cross-Clamp (min) & $29 \pm 22$ & $41 \pm 20$ & $57 \pm 22$ & $76 \pm 25$ & $\leq 0.001$ \\
\hline No. of Grafts & $1.91 \pm 0.29$ & $2.77 \pm 0.42$ & $3.79 \pm 0.41$ & $4.87 \pm 0.58$ & $\leq 0.001$ \\
\hline Venous & $0.62 \pm 0.49$ & $1.36 \pm 0.69$ & $2.21 \pm 0.78$ & $2.88 \pm 1.03$ & $\leq 0.001$ \\
\hline Arterial & $1.29 \pm 0.46$ & $1.41 \pm 0.58$ & $1.58 \pm 0.70$ & $2.00 \pm 0.98$ & $\leq 0.001$ \\
\hline Internal Thoracic Artery & $1.03 \pm 0.19$ & $1.03 \pm 0.19$ & $1.04 \pm 0.22$ & $1.06 \pm 0.27$ & 0.001 \\
\hline
\end{tabular}


*ANOVA across 4 treatment groups (overall), "chi square across 4 treatment groups (overall), $\mathrm{PCl}$ : Percutaneous Intervention, COPD: Chronic Obstructive Pulmonary Disease. 


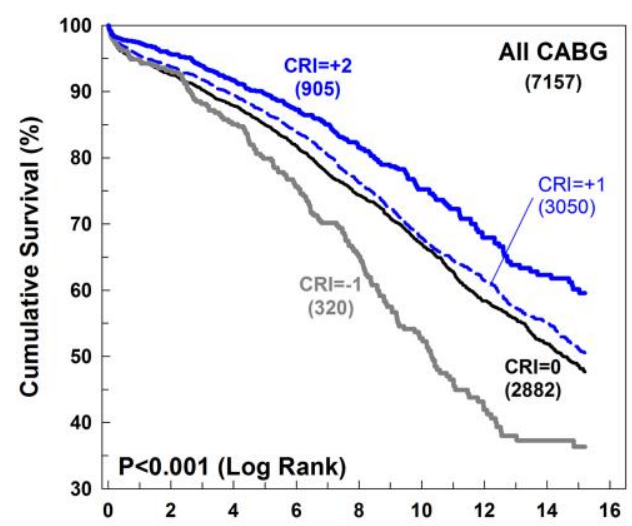

\begin{tabular}{|c|c|c|c|c|c|c|c|c|c|}
\hline \multirow{2}{*}{ Group } & \multicolumn{2}{|c|}{ Events } & \multicolumn{7}{|c|}{ Patients At Risk (n) } \\
\hline & $n$ & $\%$ & 2-yrs & 4-yrs & 6 -yrs & 8-yrs & 10-yrs & 12 -yrs & 14 yrs \\
\hline CRI $=-1$ & 160 & $56.7 \%$ & 279 & 247 & 211 & 165 & 113 & 66 & 47 \\
\hline CR $=0$ & 1095 & $38.0 \%$ & 2610 & 2427 & 2131 & 1684 & 1263 & 846 & 485 \\
\hline$C R=+1$ & 989 & $32.4 \%$ & 2791 & 2610 & 2242 & 1656 & 1042 & 679 & 424 \\
\hline$C R I=+2$ & 214 & $23.6 \%$ & 828 & 774 & 680 & 482 & 265 & 160 & 117 \\
\hline
\end{tabular}

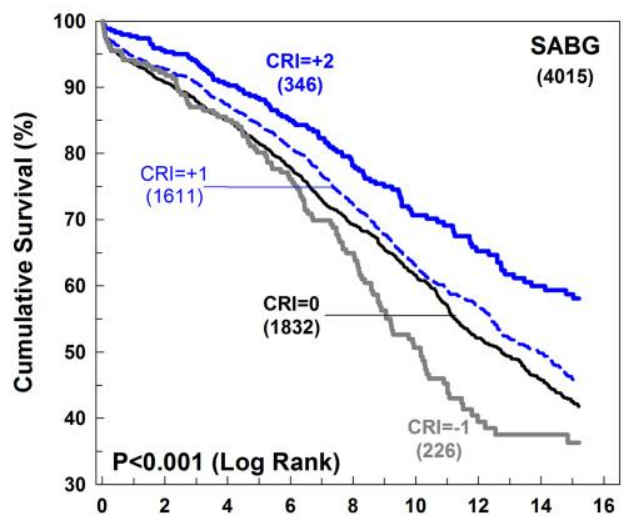

SABG ( $N=4015)$

\begin{tabular}{|l|c|c|c|c|c|c|c|c|c|}
\hline \multirow{2}{*}{ Group } & \multicolumn{2}{|c|}{ Events } & \multicolumn{7}{|c|}{ Patients At Risk (n) } \\
\cline { 2 - 11 } & $\mathbf{n}$ & $\%$ & 2-yrs & 4-yrs & 6-yrs & 8-yrs & $\mathbf{1 0 - y r s}$ & 12-yrs & 14-yrs \\
\hline CRI= -1 & $\mathbf{1 1 4}$ & $\mathbf{5 0 . 4 \%}$ & 195 & 174 & 149 & 115 & 76 & 41 & 34 \\
\hline CRI= 0 & $\mathbf{8 3 7}$ & $\mathbf{4 5 . 7 \%}$ & 1653 & 1520 & 1335 & 1042 & 793 & 532 & 352 \\
\hline CRI= +1 & $\mathbf{6 5 7}$ & $\mathbf{4 0 . 8 \%}$ & 1483 & 1371 & 1188 & 916 & 632 & 450 & 327 \\
\hline CRI= +2 & $\mathbf{1 1 0}$ & $\mathbf{3 1 . 8} \%$ & 322 & 299 & 263 & 214 & 149 & 113 & 99 \\
\hline
\end{tabular}

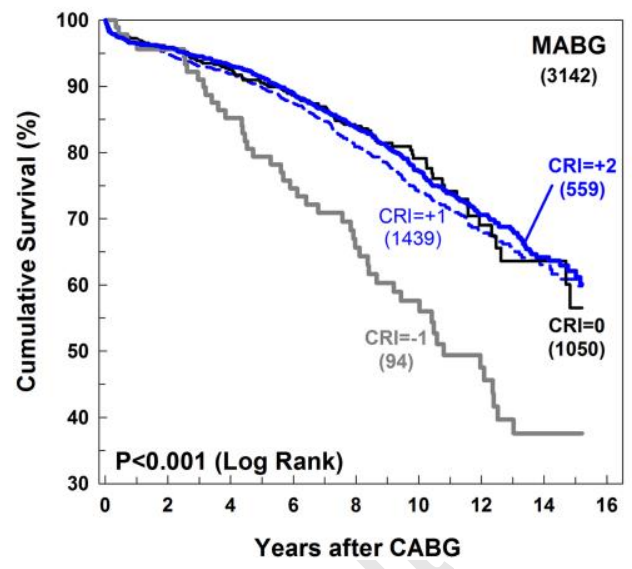

MABG ( $\mathrm{N}=3142)$

\begin{tabular}{|l|c|c|c|c|c|c|c|c|c|}
\hline \multirow{2}{*}{ Group } & \multicolumn{2}{|c|}{ Events } & \multicolumn{7}{|c|}{ Patients At Risk (n) } \\
\cline { 2 - 11 } & $\mathbf{n}$ & $\%$ & 2-yrs & 4-yrs & 6-yrs & $\mathbf{8 - y r s}$ & $\mathbf{1 0}$-yrs & $\mathbf{1 2}$-yrs & 14-yrs \\
\hline CRI= -1 & $\mathbf{4 6}$ & $\mathbf{4 8 . 9 \%}$ & 84 & 73 & 62 & 50 & 37 & 25 & 13 \\
\hline CRI= 0 & $\mathbf{2 5 8}$ & $\mathbf{2 4 . 6 \%}$ & 957 & 907 & 796 & 642 & 470 & 314 & 133 \\
\hline CRI= +1 & $\mathbf{3 3 2}$ & $\mathbf{2 3 . 1} \%$ & 1308 & 1239 & 1054 & 740 & 410 & 229 & 97 \\
\hline CRI= +2 & $\mathbf{1 0 4}$ & $\mathbf{1 8 . 6 \%}$ & 506 & 475 & 417 & 268 & 116 & 47 & 18 \\
\hline
\end{tabular}

Figure 1 


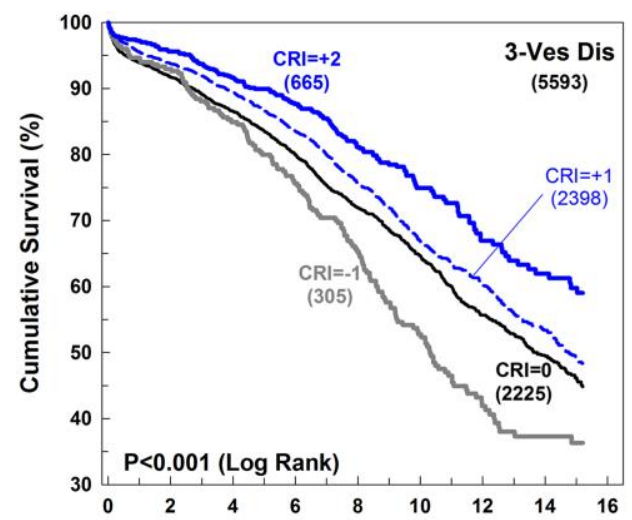

3-Ves Dis (N=5593)
\begin{tabular}{|l|c|c|c|c|c|c|c|c|c|}
\hline \multirow{2}{*}{ Group } & Events & \multicolumn{7}{|c|}{ Patients At Risk (n) } \\
\cline { 2 - 10 } & $\mathbf{n}$ & $\%$ & 2-yrs & 4-yrs & 6-yrs & 8-yrs & $\mathbf{1 0}$-yrs & 12-yrs & 14-yrs \\
\hline CRI $=\mathbf{- 1}$ & 157 & $51.5 \%$ & 269 & 240 & 206 & 163 & 112 & 66 & 47 \\
\hline CRI $=0$ & 892 & $40.1 \%$ & 2000 & 1850 & 1608 & 1242 & 921 & 599 & 341 \\
\hline CRI $=+1$ & 793 & $33.1 \%$ & 2196 & 2054 & 1754 & 1274 & 771 & 500 & 313 \\
\hline CRI $=+2$ & 160 & $\mathbf{2 4 . 1 \%}$ & 618 & 581 & 511 & 350 & 189 & 121 & 88 \\
\hline
\end{tabular}

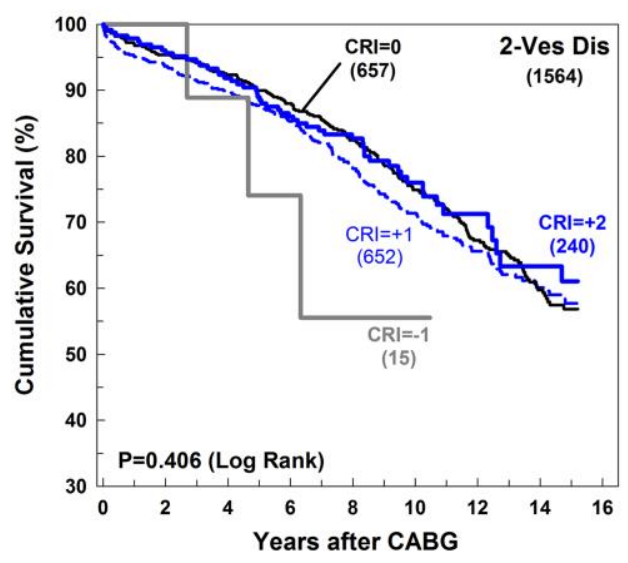

2-Ves Dis ( $\mathrm{N}=1564$ )

\begin{tabular}{|l|c|c|c|c|c|c|c|c|c|}
\hline \multirow{2}{*}{ Group } & \multicolumn{2}{|c|}{ Events } & \multicolumn{7}{|c|}{ Patients At Risk (n) } \\
\cline { 2 - 11 } & $\mathbf{n}$ & $\%$ & 2-yrs & 4-yrs & 6-yrs & 8-yrs & 10-yrs & 12-yrs & 14yrs \\
\hline CRI= -1 & 3 & $20.0 \%$ & 10 & 7 & 5 & 2 & 1 & 0 & 0 \\
\hline CRI= 0 & 203 & $30.9 \%$ & 610 & 577 & 523 & 442 & 342 & 247 & 144 \\
\hline CRI = +1 & 196 & $30.1 \%$ & 595 & 556 & 488 & 382 & 271 & 179 & 111 \\
\hline CRI $=+2$ & 54 & $22.5 \%$ & 210 & 193 & 169 & 132 & 76 & 39 & 29 \\
\hline
\end{tabular}

Figure 2 

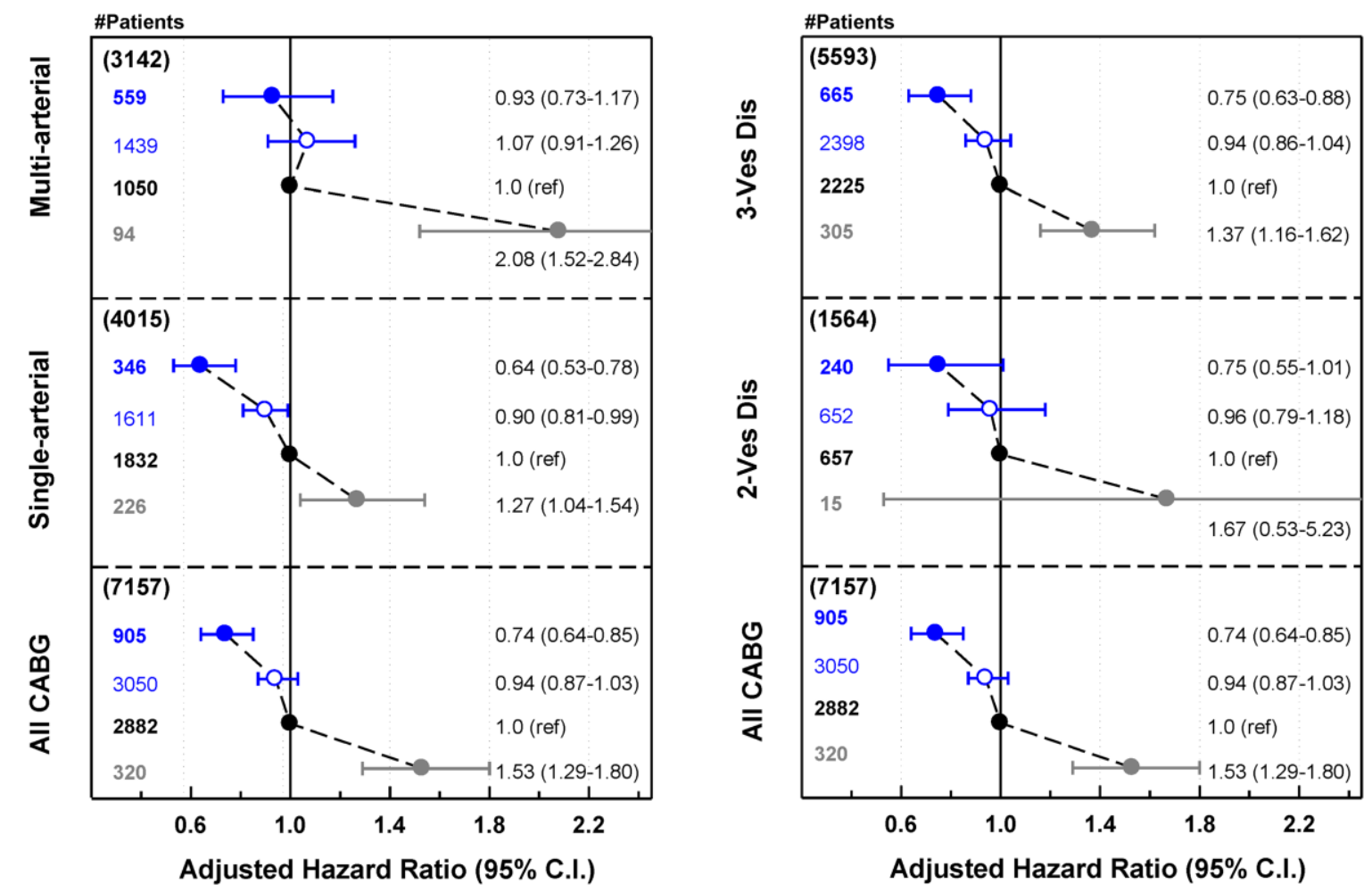

$\begin{array}{llll}-\bullet- & C R I=-1 & -O- & C R I=+1 \\ -\bullet- & C R I=0 & -\bullet- & C R I>+2\end{array}$

Figure 3 